\title{
Zosteric acid and salicylic acid bound to a low density polyethylene surface successfully control bacterial biofilm formation
}

\section{Author: C. Catto, Garth James, F. Villa, S. Villa, and F. Cappitelli}

This is an Accepted Manuscript of an article published in Pest Management Science on [date of publication], available online: http://www.tandfonline.com/10.1002/ps.5043.

Catto, C., Garth James, F. Villa, S. Villa, and F. Cappitelli. "Zosteric acid and salicylic acid bound to a low density polyethylene surface successfully control bacterial biofilm formation." Biofouling 34, no. 4 (April 2018): 440-452. DOI:10.1080/08927014.2018.1462342. 


\title{
Zosteric acid and salicylic acid bound to a low density polyethylene surface successfully control bacterial biofilm formation
}

\author{
C. Cattòn ${ }^{a, b}$ (iD) , G. James ${ }^{b}$, F. Villa ${ }^{a}$ (D) , S. Villac (iD) and F. Cappitellia iD

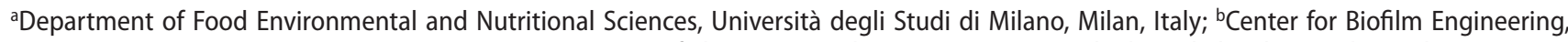 \\ Montana State University, Bozeman, MT, USA; 'Department of Pharmaceutical Sciences, Università degli Studi di Milano, Milan, Italy
}

\begin{abstract}
The active moieties of the anti-biofilm natural compounds zosteric (ZA) and salicylic (SA) acids have been covalently immobilized on a low density polyethylene (LDPE) surface. The grafting procedure provided new non-toxic eco-friendly materials (LDPE-CA and LDPE-SA) with anti-biofilm properties superior to the conventional biocide-based approaches and with features suitable for applications in challenging fields where the use of antimicrobial agents is limited. Microbiological investigation proved that LDPE-CA and LDPE-SA: (1) reduced Escherichia coli biofilm biomass by up to $61 \%$ with a mechanism that did not affect bacterial viability; (2) significantly affected biofilm morphology, decreasing biofilm thickness, roughness, substratum coverage, cell and matrix polysaccharide bio-volumes by $>80 \%$ and increasing the surface to bio-volume ratio; (3) made the biofilm more susceptible to ampicillin and ethanol. Since no molecules were leached from the surface, they remained constantly effective and below the lethal level; therefore, the risk of inducing resistance was minimized.
\end{abstract}

\section{Introduction}

The main strategy for preventing biofilm-mediated damage in medical and industrial settings relies on routine cleaning and disinfection of microbial contact surfaces (Simões et al. 2010). Unfortunately, these approaches are not universally effective because sessile microorganisms display increased tolerance to conventional antimicrobial agents (Hall-Stoodley et al. 2004). In addition, resistance towards many antibiotics has emerged in many pathogenic microbial taxa (Sousa et al. 2014; Ventola 2015).

The modification of surface properties by incorporating disinfectants, antiseptics, antibiotics and metallic nanoparticles into polymeric materials has been proposed as a promising strategy to tackle the current challenge in controlling biofilm growth (Coenye et al. 2011; Chen et al. 2013; Lo et al. 2014; Ahire et al. 2016). However, despite some of these materials being commercially available and already used in several applications, their real efficacy and recurrent drawbacks have made their use questionable (Chen et al. 2013; Pechook et al. 2015). Most of the biocide-releasing materials have a short-term efficacy, typically no longer than $24 \mathrm{~h}$, which make them less suitable for longer applications (von Eiff et al. 2005). Moreover, most of these materials exhibit a discontinuous release rate with an initially high release followed by an exponential decrease, that favors the development of antimicrobial resistance (Gharbi et al. 2012). Finally, coating materials often undergo chemical damage with a loss of efficacy (Ghosh et al. 2012; Pechook et al. 2015; Sobieh et al. 2016).

Recently, the natural compounds zosteric acid (ZA) (or $p$-(sulfoxy)cinnamic acid) and salicylic acid (SA) have been proposed as an alternative or integrative antimicrobial-free strategy to prevent biofilm development (Villa et al. 2010; Cattò et al. 2017). Instead of killing cells, ZA and SA influence the multicellular behavior of microorganisms, ie microbial adhesion, cell-to-cell communication signals and dispersion, massively decreasing biofilm development without imposing a selective pressure, thus limiting drug-resistance development (Polo et al. 2014). In previous studies by these authors, the structural requirements of ZA and SA necessary for biofilm inhibition as well as functional groups that could be exploited for their covalent linkage to an abiotic surface have been identified (Cattò et al. 2015, 2017). These works revealed that the cinnamic acid moiety is responsible for the ZA anti-biofilm 
activity. Moreover, the introduction of an amino group in the para position of the cinnamic acid moiety of ZA and on the phenyl ring of SA does not affect their anti-biofilm activity and could be exploited for their covalent linkage to a polymeric support. In the light of that information, the cinnamic acid active moiety of ZA and SA were subsequently covalently immobilized on a polyethylene surface by Dell'Orto et al. (2017) and oriented to externally exhibit their active scaffold. Since no molecules were leached from the surface, their concentration remained constantly effective and below the lethal level, reducing the risk of developing resistant strains. Although a detailed chemical study has previously been performed, further development of this technology requires a deeper microbiological investigation. In this work, the new bioengineered materials were investigated in-depth to assess their anti-biofilm performance by using a laboratory model system to simulate conditions encountered in vivo. Moreover, the synergistic effect of these bio-hybrid materials coupled with traditional antimicrobial agents was investigated.

\section{Materials and methods}

\section{Polymeric surface preparation}

Low density polyethylene (LDPE) coupons (round in shape, $d=1.27 \mathrm{~cm}$ ) were functionalized with ZA and SA derivatives according to Dell'Orto et al. (2017). Briefly, the LDPE surface was activated with a low pressure oxygen plasma treatment and graft-polymerized with 2-hydroxyethyl methacrylate (LDPE-OH). The terminal hydroxyl groups of HEMA side chains were converted into the carboxylic acid derivatives (LDPE-COOH) by treatment with succinic anhydride.

The cinnamic acid active moiety of ZA and SA has been modified by the insertion of an amino group in the para position of phenyl ring to give $p$-amino-cinnamic acid and $p$-amino-salicylic acid. Finally, a condensation reaction between the LDPE-COOH surface and the amino group was performed (LDPE-CA and LDPE-SA).

\section{Escherichia coli strain and growth conditions}

E. coli strain MG1655 was used as a model system for bacterial biofilms, being a cosmopolitan bacterium that shares a core set of genes with clinically relevant serotypes and foodborne pathogenic strains, including genes involved in biofilm formation (Faucher and Charette 2015). The strain was stored at $-80^{\circ} \mathrm{C}$ in suspensions containing $20 \%$ glycerol and $2 \%$ peptone, and was routinely grown in Luria-Bertani broth (LB, Sigma-Aldrich, St Louis, MO, USA) at $37^{\circ} \mathrm{C}$.

\section{Biofilm growth in the $\mathrm{CDC}$ reactor}

E. coli biofilm was grown on non-functionalized (LDPE, LDPE-OH and LDPE-COOH) and functionalized (LDPE-CA and LDPE-SA) coupons in the Center for Disease Control biofilm reactor (CDC reactor, BioSurface Technologies, Bozeman, MT, USA) according to Cattò et al. (2017). Briefly, the bioreactor was inoculated with $400 \mathrm{ml}$ of sterile LB medium with the addition of $1 \mathrm{ml}$ of diluted pre-washed overnight culture containing $10^{7}$ cells of $E$. coli. The culture was grown at $37^{\circ} \mathrm{C}$ with continuous stirring for $24 \mathrm{~h}$. When the 24 -h adhesion phase was over, the peristaltic pump was started and sterile $10 \%$ LB medium was continuously pumped into the reactor at a rate of $8.3 \mathrm{ml} \mathrm{min}^{-1}$. After a dynamic phase of $48 \mathrm{~h}$, functionalized and non-functionalized coupons were removed, gently washed with phosphate buffered saline (PBS, $0.01 \mathrm{M}$ phosphate buffer, $0.0027 \mathrm{M}$ potassium chloride $\mathrm{pH}$ 7.4) and processed for analysis.

\section{Plate count viability assay}

Collected coupons were transferred to $5 \mathrm{ml}$ of PBS and sessile cells were removed from the coupon surface by vortex mixing for $30 \mathrm{~s}$ and sonication for $2 \mathrm{~min}$ (Branson 3510, Branson Ultrasonic Corporation, Dunburry, CT, USA) followed by vortex mixing for another $30 \mathrm{~s}$. Serial dilutions of the resulting cell suspensions were plated on tryptic soy agar (TSA, Fisher Scientific, Hampton, NH, USA) and incubated overnight at $37^{\circ} \mathrm{C}$. Colony forming units (CFUs) were determined by the standard colony counting method. The data obtained were reported as number of viable bacterial cells normalized to the area and means were calculated. The efficacy of the anti-biofilm material was calculated as percentage reduction of the CFUs in respect to the LDPE control. Four coupons for each surface were analyzed. The experiment was repeated four times for a total of 16 coupons analyzed.

\section{Epifluorescence microscopy analysis}

The percentage of live and dead cells in the biofilm biomass grown on both non-functionalized and functionalized coupons was determined using the Live/Dead BacLight viability kit (L7012, Molecular Probes-Life Technologies, Eugene, OR, USA). Biofilm was incubated with $2 \mu \mathrm{l}$ of each fluorescent probe per $\mathrm{ml}$ of sterile filtered PBS at room temperature in the dark for $25 \mathrm{~min}$ and then rinsed with sterile PBS, according to the manufacturer's instruction. Coupons without biofilm were also stained with the dyes in order to exclude any false positive signals. Biofilm samples were visualized using a Nikon Eclipse 
E800 (Tokyo, Japan) epifluorescent microscope with excitation at $480 \mathrm{~nm}$ and emission at $516 \mathrm{~nm}$ for the green channel and excitation at $581 \mathrm{~nm}$ and emission at $644 \mathrm{~nm}$ for the red channel. Images were captured with a $60 \times, 1.0$ Numerical Aperture (NA) water immersion objective and analyzed via MetaMorph 7.5 software (Molecular Devices, Sunnyvale, CA, USA). The percentage area of stained cells was obtained by calculating at least 10 random images for each sample. The efficacy of the anti-biofilm material was calculated as the percentage reduction in the stained cells area in respect to the LDPE control images. Relative viability within the biofilm was determined by dividing the percentage area of live cells by the percentage area of dead cells in each sample. Four coupons of each surface were analyzed in each experiment. The experiment was repeated four times for a total of 16 coupons analyzed.

\section{Confocal laser scanning microscopy (CLSM) analysis}

The 3-D morphology of biofilm growth on non-functionalized and functionalized surfaces was analyzed by CLSM. Biofilm was stained with $200 \mu \mathrm{g} \mathrm{ml}^{-1}$ the of lectin concanavalin A-Texas Red conjugate dye (C825, Molecular Probes-Life Technologies) to visualize the polysaccharide component of the extracellular polymeric substances (EPS) and 1:1,000 SYBR green I fluorescent nucleic acid dye (S7563, Molecular Probes-Life Technologies) to display biofilm cells, in the dark for $30 \mathrm{~min}$. Coupons without biofilm were also stained in order to exclude any false positive signals. Biofilm samples were visualized using a Leica (Wetzlar, Germany) SP5 CLSM with excitation at $488 \mathrm{~nm}$, and emission < $530 \mathrm{~nm}$ (green channel). Images were captured with a $63 \times, 0.9$ NA water immersion objective and projections and 3-D reconstructed images of biofilm were generated using the Imaris software package (Bitplane Scientific Software, Zurich, Switzerland).

Quantitative biofilm structural parameters were calculated, including (1) mean thickness, which identifies the mean distance from the substratum in the direction normal to the substratum where there is biofilm; (2) roughness, a quantity calculated from the thickness distribution and which describes the heterogeneity of the biofilm; (3) substratum coverage, the percentage of substratum area occupied by the biofilm; (4) surface-to-volume ratio, which reflects the fraction of the biofilm area that is exposed to the nutrients; and (5) bio-volume, of the cell polysaccharide matrix, which provides an estimation of the biomass in the biofilm (Chang et al. 2015). Biofilm morphological parameters were obtained via MetaMorph 7.5 (Molecular Devices) and COMSTAT software from at least five random images for each sample according to Heydorn et al. (2000). Four coupons of each surface were analyzed. The experiment was repeated four times for a total of 16 coupons analyzed.

\section{Antimicrobial susceptibility test}

Non-functionalized and functionalized coupons with pregrown biofilm were removed from the $\mathrm{CDC}$ reactor and independently soaked in $15 \mathrm{ml}$ of $100 \mu \mathrm{g} \mathrm{ml}^{-1}$ ampicillin (Amp, BP1760-5, Fisher Scientific) for $24 \mathrm{~h}$ and $20 \%$ ethanol for $20 \mathrm{~min}$. Negative controls were set up by soaking coupons with pre-grown biofilm in $15 \mathrm{ml}$ of PBS for $24 \mathrm{~h}$ or $20 \mathrm{~min}$. The concentrations and the incubation time were chosen to mimic antibiotic therapies and daily disinfection procedures with ethanol in hospital and industrial settings. After the treatment, coupons were left in PBS for $10 \mathrm{~min}$ in order to neutralize the antimicrobial agent. Cells in the biofilm were dislodged from the coupon and collected in PBS for colony counting, as previously reported in the 'plate count viability assay section. Cells dispersed in the bulk liquid were washed by centrifugation at $8,000 \mathrm{~g}$ for $30 \mathrm{~min}$ and suspended in PBS for colony counting. The efficacy of both ampicillin and ethanol treatments was reported as the percentage reduction in respect to the LDPE control treated with PBS. For each experiment, four coupons of each surface were analyzed. The experiment was repeated four times for a total of 16 coupons analyzed.

The antimicrobial activity of $20 \%$ ethanol on E. coli biofilm pre-grown on non-functionalized and functionalized coupons was further investigated by using a CLSM method that enables the direct and real-time visualization of cell inactivation within the biofilm structure (Davison et al. 2010). The imaging technique was based on monitoring the loss of fluorescence that corresponds to the leakage of a fluorophore out of cells due to the membrane permeabilization by the biocides (Davison et al. 2010). The experiment was carried out using the FC 270 flow cell apparatus (BioSurface Technologies), according to the manufacturer's recommendations. Biofilms pre-grown on the coupons were stained with $5 \mathrm{mM}$ of syto-9 green fluorescent nucleic acid stain solution (S-34854, Molecular Probes-Life Technologies) in PBS at room temperature in the dark for $30 \mathrm{~min}$ and then rinsed with PBS. A solution of $20 \%$ ethanol was pumped through the flow cells at a rate of $1 \mathrm{ml} \mathrm{min}^{-1}$ for $20 \mathrm{~min}$. The spatiotemporal decrease in fluorescence intensity was observed using a Leica SP5 CLSM with excitation at $488 \mathrm{~nm}$, and emission $<530 \mathrm{~nm}$ (green channel) and a 63×, 0.9 NA water immersion objective. Images were analyzed by Imaris (Bitplane Scientific Software) according to Davison et al. (2010). The average green fluorescence intensity was measured at different locations within the biofilm: at the interface with the bulk fluid (top), the intermediate location (center) and the 
Table 1. Biomass within the biofilm grown on non-functionalized (LDPE, LDPE-OH, LDPE-COOH) and functionalized polyethylene surfaces (LDPE-CA, LDPE-SA) by plate count viability assay and epifluorescence analysis.

\begin{tabular}{|c|c|c|c|c|}
\hline \multirow[b]{2}{*}{ Surface } & \multirow{2}{*}{$\begin{array}{c}\text { Plate count assay } \\
\begin{array}{c}\text { Viable adhered cells } \\
\left(\mathrm{CFU} \mathrm{cm} \mathrm{cm}^{-2}\right)\end{array}\end{array}$} & \multicolumn{3}{|c|}{ Epifluorescence microscope analysis } \\
\hline & & Live cells (\%) & Dead cells (\%) & Relative viability \\
\hline LDPE & $(1.05 \pm 0.15) \times 10^{7 a}$ & $59.82 \pm 4.86^{\mathrm{a}}$ & $6.51 \pm 0.81^{a}$ & $8.48 \pm 0.34^{a}$ \\
\hline LDPE-OH & $(1.05 \pm 0.10) \times 10^{7 a}$ & $55.79 \pm 4.24^{\mathrm{a}}$ & $7.05 \pm 0.42^{\mathrm{a}}$ & $7.84 \pm 0.75^{a}$ \\
\hline LDPE-COOH & $(1.03 \pm 0.17) \times 10^{7 a}$ & $59.19 \pm 2.19^{a}$ & $7.07 \pm 0.44^{\mathrm{a}}$ & $7.37 \pm 1.24^{\mathrm{a}}$ \\
\hline LDPE-CA & $(0.42 \pm 0.08) \times 10^{7 b}$ & $25.91 \pm 4.75^{b}$ & $5.98 \pm 1.21^{\mathrm{a}}$ & $4.77 \pm 1.22^{b}$ \\
\hline LDPE-SA & $(0.40 \pm 0.07) \times 10^{7 b}$ & $17.61 \pm 3.34^{b}$ & $6.81 \pm 0.70^{\mathrm{a}}$ & $2.68 \pm 0.62^{c}$ \\
\hline
\end{tabular}

Notes: Data represent the means \pm SD of four independent measurements. Different superscript letters indicate significant differences (Tukey's HSD, $p \leq 0.05$ ) between the means of different surfaces.

interface with the coupon (bottom). Changes in intensity were normalized by the initial intensity at the beginning of the 20-min antimicrobial treatment. This normalized intensity was used to compare values of relative loss of fluorescence during 20-min biocide treatment periods. The kinetics of fluorescence loss was quantified by the parameter $\mathrm{T}_{50}$, the time elapsed from the initiation of treatment until the fluorescence intensity falls to half of its initial value at a particular spot. Three coupons of each surface were analyzed. The experiment was repeated three times for a total of nine coupons analyzed.

\section{Statistical analysis}

Two-tailed ANOVA analysis, via a software run in MATLAB environment (Version 7.0, The MathWorks Inc., Natick, MA, USA), was applied to statistically evaluate any significant differences among the samples and concentrations. The ANOVA analysis was carried out after verifying data independence (Pearson's chi-square test), normal distribution (D’Agostino-Pearson normality test) and homogeneity of variances (Bartlett's test). Tukey's honestly significant different test (HSD) was used for pairwise comparison to determine the significance of the data. Statistically significant results were depicted by $p$-values $<0.05$.

\section{Results}

\section{LDPE-CA and LDPE-SA reduce biofilm biomass without affecting cell viability}

Experiments showed that LDPE-CA and LDPE-SA had an optimal anti-biofilm performance, reducing viable adhered cells by $61.6 \pm 10.5 \%$ and $60.0 \pm 10.6 \%$ respectively in comparison to the LDPE control surface (Table 1). No significant differences were detected in viable adhered cells among the LDPE control and the LDPE-OH and LDPE-COOH linker samples (Table 1).

Epifluorescence microscopy was additionally used to provide image analysis and in situ quantification of bacterial cells. Figure 1 shows direct microscopic visualizations of the total biofilm biomass on functionalized and non-functionalized coupons and stained for live and dead cells. Microscope assay revealed that differences in dead cell data were not statistically relevant (Table 1 and Figure 1A). Conversely, biofilms on LDPE-CA and LDPE-SA showed a significant decrease in the number of live cells, corresponding to $56.7 \pm 11 \%$ and $70.6 \pm 7.3 \%$ in respect to the LDPE control sample (Table 1, Figure 1B and $\mathrm{C}$ ), confirming the results obtained in the plate count viability assay. No significant differences were detected in the live cell data between the LDPE and the LDPE-OH and LDPE-COOH linker samples (Table 1).

Coupons without biofilm and stained with the same dye did not produce detectable fluorescence and therefore no false positive signals were produced.

\section{LDPE-CA and LDPE-SA affect biofilm morphology}

Projection analysis as well as 3-D reconstructed CLSM images showed a complex biofilm on LDPE, LDPE-OH, LDPE-COOH, with an intense red and green signal corresponding to multi-layers of cells organized in macro-colonies inside a well-structured polysaccharide matrix (Figure $2 \mathrm{~A}$ and $\mathrm{B})$. Conversely, biofilm growth on LDPE-CA and LDPE-SA resulted in a significant decrease in thickness with a monolayer of dispersed cells (green signal) and very low presence of a polysaccharide matrix (red signal) (Figure 2C-F). Coupons without biofilm and stained with the same dye did not produce detectable fluorescence; therefore false positive signals were not produced.

Biofilms on both the functionalized surfaces displayed a thickness below $5 \mu \mathrm{m}$ with a decrease up to $84.5 \pm 1.2 \%$ (Table 2). Conversely, biofilm on the LDPE control surface reached about $25 \mu \mathrm{m}$ thick. Additionally, the LDPE-CA and LDPE-SA biofilm roughness significantly decreased (up to $57.0 \pm 3.9 \%$ ), indicating a uniform biofilm layer, in contrast with the patchy and more heterogeneous control biofilm. On LDPE-CA and LPDE-SA, the substratum coverage by biofilm was notably lower (up to $84.5 \pm 2.5 \%$ ) than in the corresponding non-functionalized counterpart as well as the total bio-volumes (up to $92.0 \pm 6.5 \%$ ). 

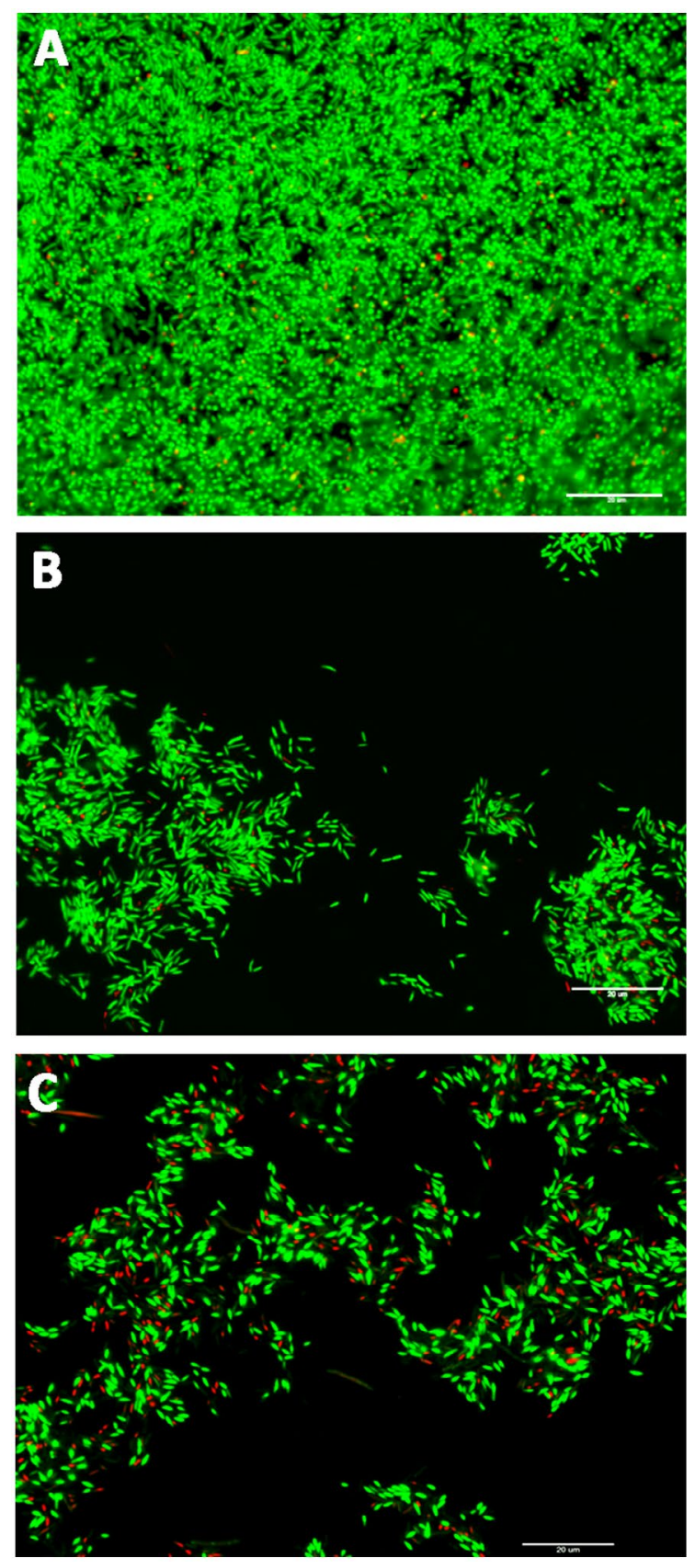

Figure 1. Representative epifluorescence microscope images of $E$. coli biofilm stained with Live/Dead BacLight viability kit and grown on non-functionalized LDPE, LDPE-OH, LDPE-COOH surfaces (A) and LDPE-CA (B) and LDPE-SA (C) functionalized surfaces $(60 \times, 1.0$ NA water immersion objective). Green fluorescence corresponds to $E$. coli live cells (excitation wavelength $\left(\lambda_{\mathrm{ex}}\right): 480 \mathrm{~nm}$ and emission wavelength $\left.\left(\lambda_{\mathrm{em}}\right): 516 \mathrm{~nm}\right)$ and red fluorescence corresponds to $E$. coli dead cells $\left(\lambda_{\mathrm{ex}}: 581 \mathrm{~nm}\right.$ and $\lambda_{\mathrm{em}}$ : $644 \mathrm{~nm})$. Scale bar $=20 \mu \mathrm{m}$.

Indeed, LDPE-CA and LDPE-SA reduced cellular (by $91.2 \pm 14 \%$ and $90.1 \pm 14 \%$ respectively) and polysaccharide matrix bio-volumes (by $99.9 \pm 11 \%$ and $94.0 \pm 17 \%$ respectively), in comparison to the LDPE control surface. The matrix/cells bio-volumes ratio displayed a value over
1 in biofilm grown on LDPE, meaning a predominance of matrix in respect to the cells. A significant reduction in matrix/cells ratio was found within the biofilm grown on LDPE-CA and LDPE-SA, with a value equal to (LDPE-SA) or less than 1 (LDPE-CA), confirming a relevant decrease in the amount of matrix. As a consequence, the biofilm exposed surface/bio-volume ratio significantly increased when biofilm was grown on LDPE-CA (16.8 \pm 0.4 -fold) and LDPE-SA (7.1 \pm 0.2 -fold). No significant differences were detected in all morphological parameters between biofilms grown on LDPE, LDPE-OH and LDPE-COOH materials.

\section{LDPE-CA and LDPE-SA enhance biofilm susceptibility toward antimicrobial agents}

To evaluate the synergistic effect of functionalized materials with traditional antimicrobial agents, biofilm pregrown on LDPE control surface and LDPE-CA and LDPE-SA was independently submitted to a treatment with $100 \mu \mathrm{g} \mathrm{ml}^{-1}$ of ampicillin and $20 \%$ ethanol.

The $24 \mathrm{~h}$ treatment with ampicillin did not significantly affect the number of viable cells in the biofilm pregrown on the LDPE surface in comparison to the biofilm grown on the same surface but treated with PBS (Table 3). Conversely, the combination of both LDPE-CA and LDPE-SA with ampicillin reduced biofilm biomass by $96.1 \pm 15 \%$ and $95.5 \pm 17 \%$, respectively, in comparison to the LDPE surface treated with PBS. Indeed, the treatment with ampicillin further decreased the number of viable cells by $37.2 \pm 6.3 \%$ and $32.0 \pm 4.9 \%$ respectively on LDPE-CA and LDPE-SA than the functionalized surfaces alone did. In the bulk liquid, no statistical differences were detected among the number of live cells from any surface after the ampicillin treatment. Interestingly, PBS alone weakly increased the number of cells dispersed from LDPE-CA and LDPE-SA biofilms.

The 20 -min treatment with $20 \%$ ethanol did not significantly affect the number of viable cells in the biofilm grown on the LDPE surface (Table 3). In contrast, the combination of both LDPE-CA and LDPE-SA with ethanol reduced biofilm biomass by $89.2 \pm 12 \%$ and $86.5 \pm 8.9 \%$, respectively, in comparison to the LDPE control surface treated with PBS. Indeed, the treatment with ethanol decreased the number of viable cells by a further $20.3 \pm 2.5 \%$ and $27.8 \pm 2.5 \%$ on LDPE-CA and LDPE-SA, respectively, compared to the functionalized surface alone. No statistical differences were detected among the number of live cells from any surface after the ethanol treatment in the bulk liquid.

Direct time-lapse CLSM analysis of the 20\% ethanol effect on a biofilm pre-grown on both LDPE control and LDPE-CA and LDPE-SA functionalized surfaces was 


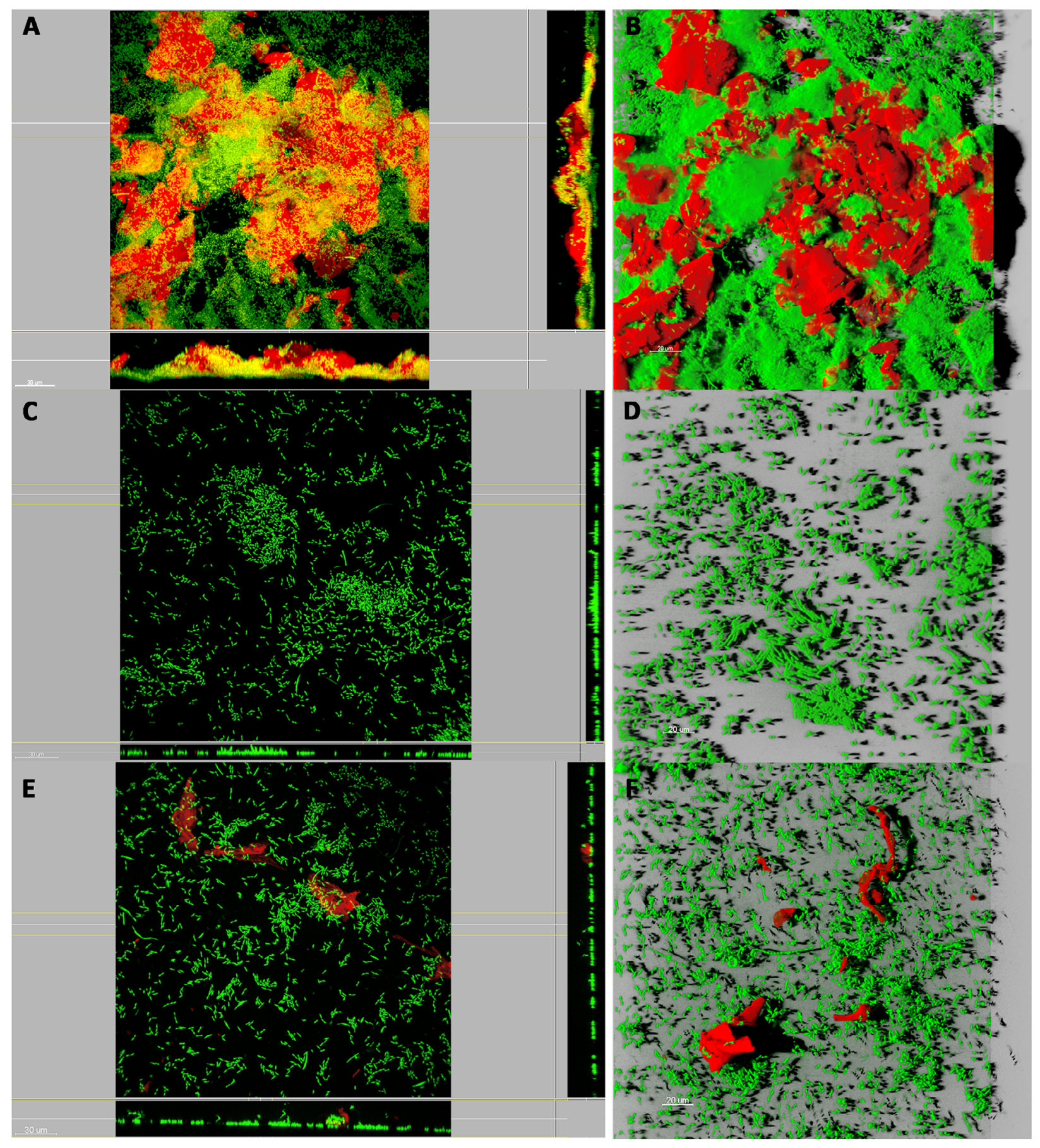

Figure 2. Representative projection analysis (column on the left) and 3-D-reconstructed CLSM images (column on the right) of $E$. coli biofilm grown on non-functionalized LDPE, LDPE-OH, LDPE-COOH surfaces (A, B), LDPE-CA (C, D) and LDPE-SA (E, F) functionalized surfaces $\left(\lambda_{\mathrm{ex}}\right.$ at $488 \mathrm{~nm}$, and $\lambda_{\mathrm{em}}<530 \mathrm{~nm}, 60 \times, 0.9 \mathrm{NA}$ water immersion objective). Live cells were stained green with SYBR green I, whereas the polysaccharide matrix was stained red with Texas Red-labeled concanavalin A. Scale bar $=20 \mu \mathrm{m}$.

additionally performed. The penetration of ethanol was inferred from the loss of green fluorescence in the biofilm during the treatment. The time-lapse images showed that the ethanol treatment did not affect the green fluorescence of the biofilm grown on the LDPE control within the $20 \mathrm{~min}$ of the experiment (Figure 3A). Moreover, no spatiotemporal changes were detected (Figure $3 \mathrm{~B}$ and C). Conversely, the ethanol treatment on biofilm grown on both LDPE-CA and LDPE-SA significantly affected the integrity of the biofilm biomass, leading to a rapid and complete loss in fluorescent intensity (Figure 3A). The highest $\mathrm{T}_{50}$ occurred at the bottom of the biofilm, at the contact with the coupon surface, and the lowest at the biofilm surface, at the interface with the bulk liquid. Indeed, on LDPE-CA, $\mathrm{T}_{50}$ values were found $10.8 \pm 1.5$-fold and $1.2 \pm 0.03$-fold higher, respectively, at the bottom and in 
Table 2. Morphological parameters of biofilms grown on non-functionalized (LDPE, LDPE-OH, LDPE-COOH) and functionalized polyethylene surfaces (LDPE-CA, LDPE-SA).

\begin{tabular}{|c|c|c|c|c|c|}
\hline & LDPE & LDPE-OH & LDPE-COOH & LDPE-CA & LDPE-SA \\
\hline Thickness $(\mu \mathrm{m})$ & $25.36 \pm 7.06^{\mathrm{a}}$ & $20.4 \pm 4.43^{\mathrm{a}}$ & $23.91 \pm 7.13^{\mathrm{a}}$ & $\begin{array}{l}3.82 \pm 1.11^{b} \\
(-84.5 \pm 1.2)\end{array}$ & $\begin{aligned} 5.00 & \pm 1.00^{b} \\
(-80.2 & \pm 1.7)\end{aligned}$ \\
\hline Roughness & $0.37 \pm 0.02^{\mathrm{a}}$ & $0.36 \pm 0.02^{\mathrm{a}}$ & $0.35 \pm 0.05^{\mathrm{a}}$ & $\begin{array}{r}0.18 \pm 0.03^{b} \\
(-51.8 \pm 10.5)\end{array}$ & $\begin{aligned} & 0.16 \pm 0.01^{b} \\
&(-57.0 \pm 3.9)\end{aligned}$ \\
\hline Substratum coverage (\%) & $68.40 \pm 5.02^{\mathrm{a}}$ & $64.44 \pm 2.49^{\mathrm{a}}$ & $62.19 \pm 1.42^{\mathrm{a}}$ & $\begin{array}{l}14.37 \pm 3.83^{b} \\
(-78.9 \pm 5.6)\end{array}$ & $\begin{array}{l}10.62 \pm 1.72^{b} \\
(-84.5 \pm 2.5)\end{array}$ \\
\hline Surface/bio-volume $\left(\mu \mathrm{m}^{2} \mu \mathrm{m}^{-3}\right) \times 10^{-1}$ & $0.18 \pm 0.05^{\mathrm{a}}$ & $0.16 \pm 0.02^{\mathrm{a}}$ & $0.16 \pm 0.01^{\mathrm{a}}$ & $\begin{array}{c}3.08 \pm 0.10^{b} \\
(+16.8 \pm 0.4 \text {-fold })\end{array}$ & $\begin{array}{l}1.65 \pm 0.69^{c} \\
(+7.1 \pm 0.2 \text {-fold })\end{array}$ \\
\hline Total bio-volume $\left(\mu \mathrm{m}^{3} \mu \mathrm{m}^{-2}\right)$ & $91.03 \pm 12.25^{\mathrm{a}}$ & $92.51 \pm 11.66^{\mathrm{a}}$ & $90.62 \pm 9.20^{\mathrm{a}}$ & $\begin{array}{c}3.85 \pm 0.09^{b} \\
(-92.0 \pm 6.5)\end{array}$ & $\begin{array}{l}9.18 \pm 0.21^{b} \\
(-91.3 \pm 2.6)\end{array}$ \\
\hline Cell bio-volume $\left(\mu \mathrm{m}^{3} \mu \mathrm{m}^{-2}\right)$ & $34.46 \pm 7.48^{\mathrm{a}}$ & $35.59 \pm 3.64^{\mathrm{a}}$ & $32.48 \pm 4.44^{\mathrm{a}}$ & $\begin{array}{r}3.85 \pm 0.09^{b} \\
(-91.2 \pm 14.1)\end{array}$ & $\begin{array}{r}4.48 \pm 0.35^{\mathrm{b}} \\
(-90.1 \pm 13.7)\end{array}$ \\
\hline $\begin{array}{l}\text { Polysaccharide matrix bio-volume } \\
\left(\mu \mathrm{m}^{3} \mu \mathrm{m}^{-2}\right)\end{array}$ & $59.88 \pm 10.97^{a}$ & $56.91 \pm 8.02^{\mathrm{a}}$ & $58.15 \pm 4.75^{\mathrm{a}}$ & $\begin{array}{r}0.00 \pm 0.01^{\mathrm{b}} \\
(-99.9 \pm 11.3)\end{array}$ & $\begin{array}{r}3.99 \pm 1.05^{\mathrm{b}} \\
(-94.0 \pm 16.7)\end{array}$ \\
\hline Matrix/cells bio-volume ratio & $1.44 \pm 0.23^{\mathrm{a}}$ & $1.60 \pm 0.06^{\mathrm{a}}$ & $1.63 \pm 0.13^{\mathrm{a}}$ & $\begin{aligned} 0.00 & \pm 0.00^{b} \\
(-88.4 & \pm 3.0)\end{aligned}$ & $\begin{array}{l}1.00 \pm 0.13^{c} \\
(-99.9 \pm 0.1)\end{array}$ \\
\hline
\end{tabular}

Notes: In brackets, the percentage reduction/increase in comparison to the LDPE control sample. Data represent the means \pm SD of four independent measurements. Different superscript letters indicate significant differences (Tukey's HSD, $p \leq 0.05$ ) between the means of different surfaces.

Table 3. Viable adhered cells (CFU $\mathrm{cm}^{-2}$ ) on non-functionalized (LDPE) and functionalized coupons (LDPE-CA, LDPE-SA) and those released into the surrounding bulk liquid after treatment for $24 \mathrm{~h}$ with $100 \mathrm{\mu g} \mathrm{ml}^{-1}$ ampicillin or PBS and treatment for 20 min with 20\% ethanol or PBS.

\begin{tabular}{|c|c|c|c|c|}
\hline & \multicolumn{4}{|c|}{ Ampicillin (24 h) } \\
\hline & \multicolumn{2}{|c|}{ Coupon } & \multicolumn{2}{|c|}{ Bulk liquid } \\
\hline & PBS & Amp & PBS & Amp \\
\hline$\overline{\mathrm{LDPE}}$ & $(1.13 \pm 0.18) \times 10^{7 a}$ & $(9.28 \pm 3.51) \times 10^{6 a}$ & $(3.39 \pm 0.35) \times 10^{7 a}$ & $(3.57 \pm 0.92) \times 10^{6 c}$ \\
\hline LDPE-CA & $(4.05 \pm 1.07) \times 10^{6 b}$ & $(4.40 \pm 0.70) \times 10^{5 c}$ & $(4.13 \pm 0.46) \times 10^{7 b}$ & $(4.69 \pm 0.28) \times 10^{6 c}$ \\
\hline \multirow[t]{4}{*}{ LDPE-SA } & $(4.72 \pm 0.52) \times 10^{6 b}$ & $(5.12 \pm 0.91) \times 10^{5 c}$ & $(4.16 \pm 0.29) \times 10^{7 b}$ & $(3.84 \pm 0.38) \times 10^{6 c}$ \\
\hline & \multicolumn{4}{|c|}{ Ethanol (20 min) } \\
\hline & \multicolumn{2}{|c|}{ Coupon } & \multicolumn{2}{|c|}{ Bulk liquid } \\
\hline & PBS & EtOH & PBS & $\mathrm{EtOH}$ \\
\hline LDPE & $(1.07 \pm 0.13) \times 10^{7 \mathrm{a}}$ & $(1.00 \pm 0.04) \times 10^{7 \mathrm{a}}$ & $(3.39 \pm 0.34) \times 10^{7 \mathrm{a}}$ & $(1.87 \pm 0.08) \times 10^{6 b}$ \\
\hline LDPE-CA & $(3.32 \pm 0.32) \times 10^{6 b}$ & $(1.16 \pm 0.16) \times 10^{6 c}$ & $(4.13 \pm 0.60) \times 10^{7 \mathrm{a}}$ & $(5.39 \pm 0.50) \times 10^{6 b}$ \\
\hline LDPE-SA & $(4.41 \pm 0.78) \times 10^{6 b}$ & $(1.44 \pm 0.15) \times 10^{6 c}$ & $(4.16 \pm 0.51) \times 10^{7 \mathrm{a}}$ & $(9.53 \pm 0.17) \times 10^{6 b}$ \\
\hline
\end{tabular}

the center of the biofilm in respect to the top (Figure 3B and C). Also, in the biofilm on LDPE-SA $\mathrm{T}_{50}$ progressively decreased from the bottom to the center, with values respectively $40.0 \pm 1.2$-fold and $4.5 \pm 0.3$-fold higher in comparison to the top (Figure $3 \mathrm{~B}$ and $\mathrm{C}$ ). $\mathrm{T}_{50}$ at the bottom was $3.8 \pm 0.06$-fold higher in the biofilm grown on LDPE-CA in comparison to that grown on LDPE-SA, while no differences in the $\mathrm{T}_{50}$ were detected at the center and the top of the biofilm (Figure 3C).

\section{Discussion}

In this work, the functionalization of LDPE surface with biocide-free anti-biofilm compounds, ie, ZA and SA, was carried out to obtain new non-toxic materials able to hinder biofilm formation. LDPE has been chosen as the most common widespread polymer in many applications, eg biomedical devices (Siddiqa et al. 2015) and food packaging
(Raj et al. 2004), due to its excellent chemical resistance, low wetting properties in aqueous media, high impact strength, light weight and high flexibility (Sastri 2010). Notably, to date, the functionalization was performed only when polymeric material is a film, with a thickness of around a few nanometres. In this work $1.6 \mathrm{~mm}$ thickness LDPE coupons were used, opening the potential to extend the technology to other materials of a similar thickness.

In a first step, ZA and SA have been modified to be suitable for covalent grafting to the LDPE surface.

In a previous study the authors demonstrated that the cinnamic scaffold is the specific structural feature responsible for the anti-biofilm performance of ZA (Cattò et al. 2015). Moreover, these studies of the relationship between structure and anti-biofilm activity revealed that the anti-biofilm activity of ZA and its derivatives depended upon the presence of a carboxylate anion and, consequently, on its hydrogen-donating ability. In addition, the conjugated 
A
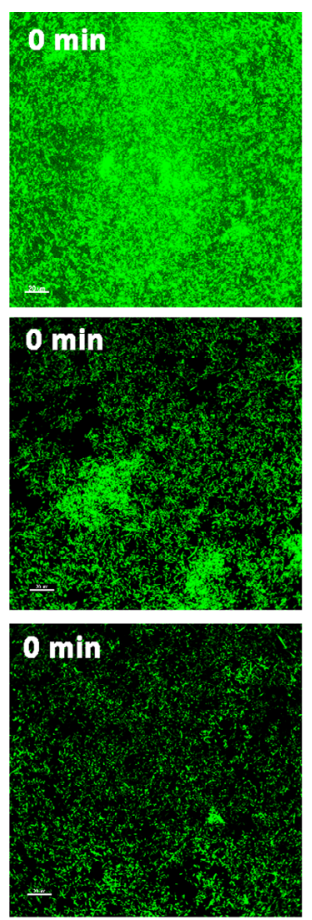

B
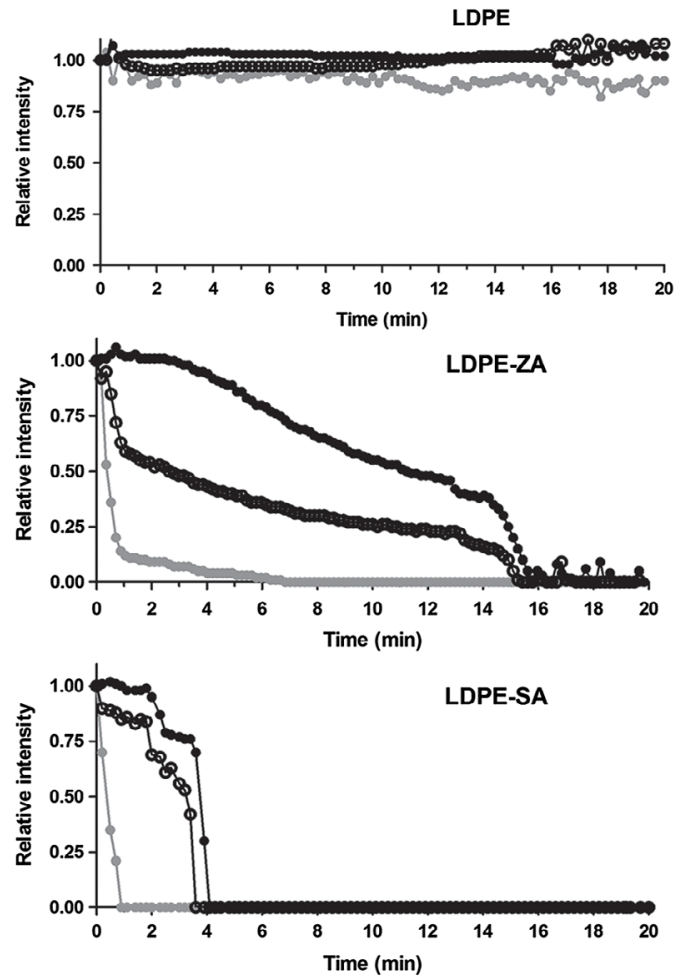
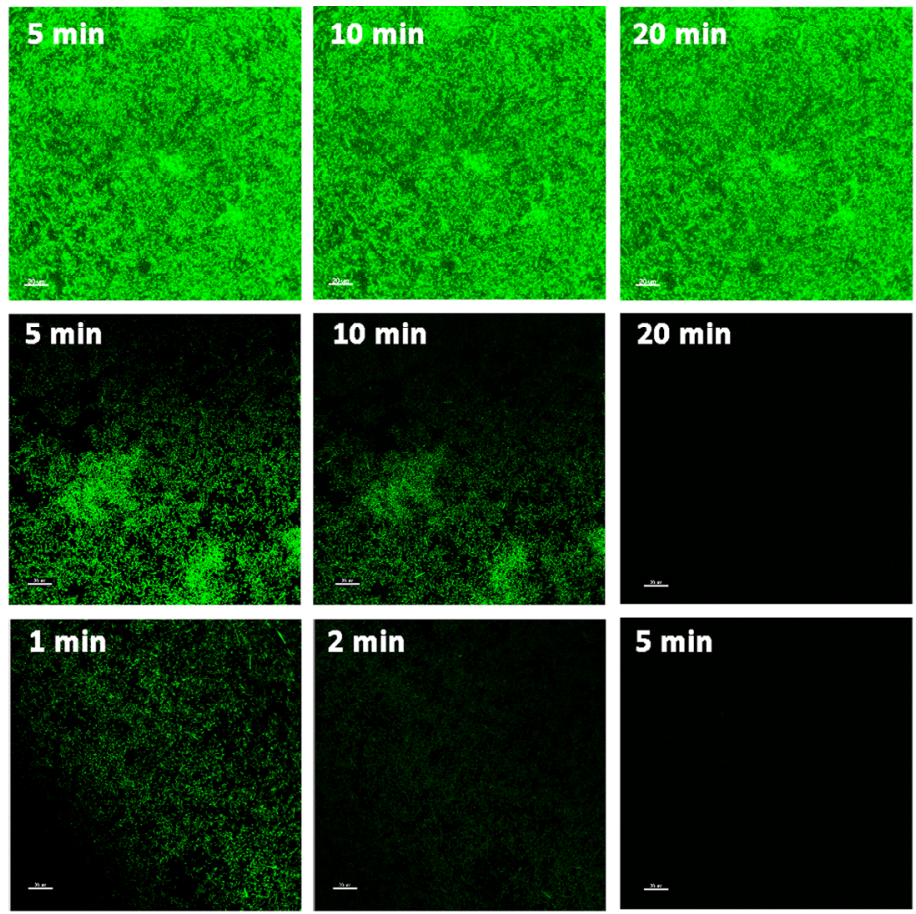

C

\begin{tabular}{lccc} 
& \multicolumn{3}{c}{$\mathrm{T}_{50}(\mathrm{~min})$} \\
\hline & Bottom & Center & Top \\
\hline LDPE & N.D. & N.D. & N.D. \\
\hline LDPE-ZA & $14.35 \pm 0.22^{\mathrm{a}}$ & $3.17 \pm 0.12^{\mathrm{b}}$ & $0.36 \pm 0.05^{\mathrm{c}}$ \\
\hline LDPE-SA & $3.75 \pm 0.10^{\mathrm{d}}$ & $3.22 \pm 0.08^{\mathrm{b}}$ & $0.35 \pm 0.05^{\mathrm{c}}$ \\
\hline
\end{tabular}

Figure 3. Time-lapse CLSM analysis of the $20 \%$ ethanol action against $E$. coli biofilm pre-grown on non-functionalized and functionalized polyethylene surfaces within the 20 min antimicrobial susceptibility test. (A) Time-lapse CLSM representative images of biofilm pregrown on LDPE control surface (first line), LDPE-CA (second line) and LDPE-SA (third line) during the treatment with 20\% ethanol. Live cells were stained green with Syto $9\left(\lambda_{\mathrm{ex}}\right.$ at $488 \mathrm{~nm}$, and $\lambda_{\mathrm{em}}<530 \mathrm{~nm}, 60 \times, 1.0$ NA water immersion objective). Scale bar $=20 \mu \mathrm{m}$. (B) Relative fluorescence intensity against time at the interface with the bulk fluid (gray symbols), at the intermediate location (open symbols) and at the interface with the coupon (black symbols). Fluorescent intensity in a specific biofilm region was normalized by the initial intensity in that same region. (C) The $_{50}$ parameter (time elapsed from the initiation of treatment until the fluorescence intensity fell to half of its initial value at a particular spot) calculated at the interface with the bulk fluid (top), at the intermediate location (center) and at the interface with the coupon (bottom). ND, not determined as fluorescence intensity did not reach $50 \%$ of the initial intensity within the experimental time. 
aromatic system was integral to the anti-biofilm activities of ZA since the presence of the double bond stabilized the carboxylate anion. In contrast, the sulfate group is not integral to the anti-biofilm activity of ZA and its presence in the ZA structure is part of an ecological phytochemical strategy to make the active cinnamic moiety more soluble and thus more mobile in the water-based sap of the vascular transport system (Baek et al. 2010). In line with the previous research, in this work ZA has been modified by replacing the sulfate group in the para position of the phenyl ring with an amino group suitable for a condensation reaction with the LDPE-COOH surface. Cattò et al. (2015) identified $p$-amino-cinnamic acid as an excellent candidate to be covalently linked to a polymeric support. Moreover, $p$-amino-cinnamic acid displayed anti-biofilm activity, suggesting that the addition of an amino group in the para position of phenyl ring of the cinnamic acid moiety does not affect the anti-biofilm performance of the molecule. Similarly, Cattò et al. (2017) demonstrated that the addition of the amino group in the para position of the SA phenyl ring does not affect its anti-biofilm performance. Moreover, docking calculations suggested that the presence of the amino group only slightly influences the binding mode to the tryptophanase TnaA targeted protein predicted for SA, with a mechanism of action that involves the same protein residues expected for SA. Thus, an amino group in the para position of phenyl ring has been added to the SA chemical structure, allowing its covalent binding with the LDPE-COOH surface.

In the past, ZA and SA were incorporated in different polymeric materials able to gradually release the anti-biofilm molecules into the surrounding area (Geiger et al. 2004; Barrios et al. 2005; Rosenberg et al. 2008; Nowatzki et al. 2012). However, in most cases these systems showed several problems such as the non-uniform distribution of the anti-biofilm compounds inside the material and the formation of aggregates due to the incomplete miscibility of the molecules in the polymers. In addition, a constant release rate of the active compound has neither been achieved nor monitored, limiting the effectiveness of the technology to only a short period of time and making this system often unfeasible for a wide range of practical applications (Barrios et al. 2005; Nowatzki et al. 2012). Here, these issues have all been addressed, as ZA and SA are uniformly distributed and oriented to externally exhibit their active scaffold. This allows the functionalized material to directly interact with bacterial cells even at the early stages of biofilm formation (Dell'Orto et al. 2017). In this work, the efficacy of the anti-biofilm materials was evaluated in depth with the aim of transferring the technology into consumer products suitable for a widespread distribution.
A laboratory standard procedure using a CDC biofilm reactor was employed to grow E. coli biofilms, simulating the conditions to which surfaces of a wide range of applications are subjected during their use (Goeres et al. 2005; Gilmore et al. 2010; Williams et al. 2011). Experiments revealed that biofilms were significantly affected when grown on both LDPE-CA and LDPE-SA in comparison to the LDPE surface. Indeed, according to the anti-biofilm ranges proposed by Cattò et al. (2015), LDPE-CA and LDPE-SA displayed optimal anti-biofilm performance, decreasing viable biomass over the $60 \%$ in comparison to the control material. Moreover, in situ stained biofilm for live and dead bacterial cells confirmed that the reduction in the biofilm biomass was achieved on both functionalized surfaces by a mechanism that did not affect bacterial viability, an important factor in the challenge to limit the risk of developing resistant microbial strains. The results are in line with those previously achieved with ZA and SA free in solution. Villa et al. (2010) found that $500 \mathrm{mg} \mathrm{l}^{-1}$ of ZA reduced $E$. coli cell adhesion up to $70 \%$ whereas Cattò et al. (2017) proved that $25 \mathrm{mg} \mathrm{l}^{-1}$ of SA inhibited E. coli biofilm by $68 \%$. In both cases the anti-biofilm performances were displayed with a mechanism more subthe than simple killing activity, modulating the activity of some ZA and SA targeted proteins and providing conditions to which the best microbial strategy is to escape from the adverse conditions, instead of persisting in the biofilm lifestyle.

Additional experiments performed by CLSM showed the massive impacts of the functionalized material on biofilm morphology. Biofilm grown on LDPE-CA and LDPE-SA showed a significant decrease in thickness and roughness with a uniform monolayer of dispersed cells and a significant lower bio-volume of polysaccharide matrix. Conversely, LDPE control images showed a complex heterogeneous biofilm, with an intense fluorescence signal corresponding to dense multi-layers of cells uniformly distributed over the substratum and organized in macro-colonies inside a well-structured robust polysaccharide matrix. Cattò et al. (2017) showed that SA free in solution significantly decreased the amount of polysaccharide in the matrix of the E. coli biofilm, while Vila and Soto (2012) hypothesized that SA plays a role in matrix production, decreasing the expression of the membrane protein, OmpA, involved in the transport of polymeric substances required for the formation of the EPS outside the cells.

The exposed biofilm surface to bio-volume ratio, which reflects the fraction of biofilm area that is exposed to the nutrient flow, was higher in the biofilm grown on the functionalized surfaces in contrast to the biofilm grown on the non-functionalized counterpart. A high surface to 
bio-volume ratio indicates the presence of small cell clusters attached to the substratum, while a low value indicates the occurrence of larger micro-colonies within the biofilm (Mangwani et al. 2016). Indeed, surface to bio-volume ratio is an indicative parameter of adaptation of the biofilm to the environment and it is reported to increase in stress conditions (Heydorn et al. 2000; Bester et al. 2011; Mangwani et al. 2016).

Experiments showed no significant differences in adhered cells, cell viability and the quantitative structural parameters of the biofilm between the LDPE control surface and the LDPE-OH and LDPE-COOH linker surfaces. These results suggest that the functionalization procedure did not introduce modification in the LDPE structure that affected cell adhesion and viability as well as biofilm morphology. Consequently, the anti-biofilm performance of LDPE-CA and LDPE-SA was totally attributable to ZA and SA covalently immobilized on the surface. Indeed, the data suggest that the ZA and SA functionalized on a LDPE surface are able to exert their anti-biofilm activity even immobilized on a surface, providing data comparable with those previously reported with the same molecules free in solution.

The combination of anti-biofilm surfaces with conventional treatments could be a step forward to maximizing the anti-biofilm performance of the polymeric materials. Surfaces that retard adhesion and consequently biofilm formation greatly enhance the efficacy of cleaning treatments and disinfection procedures. Once biofilm integrity is affected, bacteria are more susceptible to conventional antimicrobial agents than those in the intact biofilms, allowing reduction in the antimicrobial doses and providing more potent control against the development of drug-resistant strains (Cui et al. 2015; Cheesman et al. 2017).

In this study, the ability of LDPE-CA and LDPE-SA to enhance biofilm susceptibility to conventional antimicrobial agents, ie ampicillin and ethanol, has been shown. Indeed, both ampicillin and ethanol significantly further decreased the number of viable adhered cells on both functionalized materials with respect to the non-functionalized controls. CLSM analysis revealed that biofilms grown on LDPE-CA and LDPE-SA were deeply affected with an extremely low amount of polysaccharide matrix. The biofilm matrix is a barrier with the potential to reduce the penetration of antibiotics or biocides within the biofilm, either by physically slowing their diffusion or chemically reacting with them (Rabin et al. 2015). Although the involvement of other mechanisms is not excluded, biofilm grown on functionalized materials might be more susceptible to antimicrobial agents, as these compounds can penetrate more easily within the biofilm matrix, exerting their effect even at a concentration below those normally used in traditional applications.

In addition, an integrative solution by coupling a functionalized material with ethanol could have wider applications than a strategy with ampicillin. Ethanol is the most popular broad range bactericidal agent (McDonnell and Russell 1999). It is used for example in the disinfection of skin, medical equipment, and cooking equipment because it is volatile, leaves minimal residue, and is harmless, even if intraoral intake occurs. Despite its extensive and long-standing use as an antiseptic, there is no evidence of acquired microbial resistance (Balestrino et al. 2009). Importantly, ethanol is universally available without restriction and with an affordable price. Conversely, antibiotic resistance is a global issue and sooner or later, after several antibiotic treatments, ampicillin resistance naturally occurs (Ventola 2015). Moreover, antibiotic treatment is limited to only few sectors of the economy, ie the medical sector, and antibiotic use often requires a prescription. On the basis of these considerations, the spatial and temporal patterns of ethanol penetration into the biofilm pre-grown on both non-functionalized and functionalized surfaces were additionally investigated by direct time-lapse CLSM analysis. This method is suitable for mimicking antimicrobial treatments that cause membrane permeabilization, eg ethanol (Davison et al. 2010; Corbin et al. 2011). The biofilm on LDPE surface retained its initial fluorescence over the 20-min experimental test, while ethanol caused a loss of fluorescence in the biofilms pre-grown on both LDPE-CA and LDPE-SA. A distinct spatiotemporal pattern of fluorescence loss was observed for both the surfaces investigated. In both cases the antimicrobial action of ethanol occurred very rapidly at the biofilm surface and slower at the bottom of biofilm, in contact with the coupon surface. However, at the bottom of the biofilm the decrease of fluorescence intensity was $3.8 \pm 0.06$-fold more rapid in the biofilm grown on LDPE-SA in comparison to that grown on LDPE-CA, suggesting a stronger action of ethanol against biofilm on LDPE-SA rather than on LDPE-CA. The direct timelapse CLSM analysis did not give evidence of whether the treatment caused the killing or the removal of the biomass from the biofilm or both. The loss of a green color proved that cell membranes were compromised, not killed (Corbin et al. 2011). In addition, the plate count assay of cells in the bulk liquid after ethanol treatment performed in the previous experiments suggested the presence of a number of live cells.

The low toxicity of both ZA and SA are promising for the spread of this technology to different sectors of science and technology, resulting in new, safe and eco-friendly types of products suitable for applications not only in the 
more traditionally studied sector of the economy, ie the medical area, but in other challenging fields, especially when the use of antimicrobial agents is limited for safety reasons, eg in the food industry. Indeed, a food-packaging surface with immobilized anti-biofilm molecules would offer the advantage of extending the shelf life of food and preventing the growth of food-borne bacteria/pathogens at early stages, without direct addition of preservative agents, and such that only a low level of preservative comes in contact with the food. Additionally, industrial installation and pipelines, such as drinking water networks, could benefit from this research, providing safe material for water distribution systems that, at the same time, maintains high purity standard levels of water and limits the need for the less effective and expensive disinfection procedures.

Moreover, in addition to E. coli, the technology might be potentially used with a broad-spectrum activity against mixed infections. Most of the proteins targeted by ZA and SA are highly conserved with often a $100 \%$ of identity in a number of microorganisms, including pathogens that are a concern in the food processing industry and the healthcare sector and that are responsible for extensive damage to crops in the agricultural field (eg Pseudomonas spp., Klebsiella spp., Salmonella spp., Shigella spp., Candida spp., and Fusarium spp.) (Cattò et al. 2015, 2017). Additionally, ZA free in solution has been shown to be beneficial against Bacillus cereus, P. putida, Aspergillus niger, Penicillium citrinum, Candida albicans, Colletotricum lindemuthianum and Magnaporthe grisea (Stanley et al. 2002; Villa et al. 2010, 2011; Polo et al. 2014) while SA has shown activity toward $P$. aeruginosa, Klebsiella pneumoniae, Staphylococcus epidermidis, P. syringae, Pectobacterium carotovorum and C. albicans (Farber and Wolff 1993; Muller et al. 1998; Dong et al. 2012; Lagonenko et al. 2013; Cattò et al. 2017). Thus, it is likely that LDPE-CA and LDPE-SA could exert their anti-biofilm activity also against other microorganisms.

In conclusion, the active moiety of the natural compounds ZA and SA have been successfully immobilized on a LDPE surface, providing a new class of polymeric materials with extended shelf-life and anti-biofilm properties superior to the conventional biocide-based approaches in terms of addressing the problem of detrimental biofilms. Indeed, the development of materials that can resist or prevent bacterial adhesion in a non-leaching modality and with biocidal free features constitutes a promising and emerging approach to deal with material-associated biofilms and an important achievement toward the dramatic increase of antimicrobial resistance among pathogens. Moreover, the covalent grafting guarantees the material has a long life, as the molecules become permanently attached and integrated into the scaffold structure of polymers.
The work described in this study could be extended to other polymeric materials as well as natural molecules. Notably, the use of plasma technology in the grafting procedure makes each surface, including those that do not possess the required chemical features, suitable for covalent binding, without changing the material bulk.

There is much scope for further studies, following on from this pioneering work. A multitude of natural compounds have shown promising anti-biofilm properties suitable for the development of improved effective ecofriendly anti-biofilm materials (Villa et al. 2013). However, the functional groups required by these molecules to exert their anti-biofilm activity and, as a consequence, the binding site needed for their surface immobilization are poorly known, or even completely unknown.

\section{Disclosure statement}

No potential conflict of interest was reported by the authors.

\section{Funding}

This work was supported by the Fondazione Cariplo [grant number 2011-0277].

\section{ORCID}

C. Cattò (D) http://orcid.org/0000-0002-3709-1802

F. Villa (D) http://orcid.org/0000-0003-2930-4684

S. Villa (D) http://orcid.org/0000-0002-0636-7589

F. Cappitelli (D) http://orcid.org/0000-0003-1237-1813

\section{References}

Ahire JJ, Hattingh M, Neveling DP, Dicks LM. 2016. Coppercontaining anti-biofilm nanofiber scaffolds as a wound dressing material. PLoS One. 11:e0152755. doi: 10.1371/ journal.pone.0152755.

Baek D, Pathange P, Chung J-S, Jiang J, Gao L, Oikawa A, Hirai MY, Saito K, Pare PW, Shi H. 2010. A stress-inducible sulphotransferase sulphonates salicylic acid and confers pathogen resistance in Arabidopsis. Plant Cell Environ. 33:1383-1392.

Balestrino D, Souweine B, Charbonnel N, Lautrette A, Aumeran C, Traoré O, Forestier C. 2009. Eradication of microorganisms embedded in biofilm by an ethanol-based catheter lock solution. Nephrol Dial Transplant. 24:32043209.doi: 10.1093/ndt/gfp187.

Barrios CA, Xu Q, Cutright T, Newby BM. 2005. Incorporating zosteric acid into silicone coatings to achieve its slow release while reducing fresh water bacterial attachment. Colloids Surf B Biointerfaces. 41:83-93. doi: 10.1016/j. colsurfb.2004.09.009.

Bester E, Kroukamp O, Hausner M, Edwards EA, Wolfaardt GM. 2011. Biofilm form and function: carbon availability affects biofilm architecture, metabolic activity and planktonic cell yield. J Appl Microbiol. 110:387-398. doi: 10.1111/jam.2011.110.issue-2. 
Cattò C, Dell'Orto S, Villa F, Villa S, Gelain A, Vitali A, Marzano V, Baroni S, Forlani F, Cappitelli F. 2015. Unravelling the structural and molecular basis responsible for the antibiofilm activity of zosteric acid. PLoS One. 10:e0131519. doi: 10.1371/journal.pone.0131519.

Cattò C, Grazioso G, Dell'Orto S, Gelain A, Villa S, Marzano V, Vitali A, Villa F, Cappitelli F, Forlani F. 2017. The response of Escherichia coli biofilm to salicylic acid. Biofouling. 33:235251. doi: 10.1080/08927014.2017.1286649.

Chang YW, Fragkopoulos AA, Marquez SM, Kim HD, Angelini TE, Fernandez-Nieves A. 2015. Biofilm formation in geometries with different surface curvature and oxygen availability. New J Phys. 17:033017. doi: 10.1088/13672630/17/3/033017.

Cheesman MJ, Ilanko A, Blonk B, Cock IE. 2017. Developing new antimicrobial therapies: are synergistic combinations of plant extracts/compounds with conventional antibiotics the solution? Pharmacogn Rev. 11:57-72.

Chen M, Yu Q, Sun H. 2013. Novel strategies for the prevention and treatment of biofilm related infections. Int J Mol Sci. 14:18488-18501. doi: 10.3390/ijms140918488.

Coenye T, De Prijck K, Nailis H, Nelis HJ. 2011. Prevention of Candida albicans biofilm formation. Open Mycol J. 5:9-20. doi: 10.2174/1874437001105010009.

Corbin A, Pitts B, Parker A, Stewart PS. 2011. Antimicrobial penetration and efficacy in an in vitro oral biofilm model. Antimicrob Agents Chemother. 55:3338-3344. doi: 10.1128/ AAC.00206-11.

Cui JH, Ren B, Tong YJ, Dai HQ, Zhang LX. 2015. Synergistic combinations of antifungals and anti-virulence agents to fight against Candida albicans. Virulence. 6:362-371. doi: 10.1080/21505594.2015.1039885.

Davison WM, Pitts B, Stewart PS. 2010. Spatial and temporal patterns of biocide action against Staphylococcus epidermidis biofilms. Antimicrob Agents Chemother. 54:2920-2927. doi: 10.1128/AAC.01734-09.

Dell'Orto S, Cattò C, Villa F, Forlani F, Vassallo E, Morra M, Cappitelli F, Villa S, Gelain A. 2017. Low density polyethylene functionalized with antibiofilm compounds inhibits Escherichia coli cell adhesion. J Biomed Mater Res A. 105:3251-3261. doi: 10.1002/jbm.a.v105.12.

Dong Y, Huang C, Park J, Wang G. 2012. Growth inhibitory levels of salicylic acid decrease Pseudomonas aeruginosa fliC flagellin gene expression. JEMI. 16:73-78.

Farber BF, Wolff AG. 1993. Salicylic acid prevents the adherence of bacteria and yeast to silastic catheters. J Biomed Mater Res. 27:599-602. doi: 10.1002/(ISSN)1097-4636.

Faucher SP, Charette SJ. 2015. Bacterial pathogens in the nonclinical environment. Front Microbiol. 6:331.

Geiger T, Delavy P, Hany R, Schleuniger J, Zinn M. 2004. Encapsulated zosteric acid embedded in poly 3-hydroxyalkanoate coatings - protection against biofouling. Polym Bull. 52:65-72.

Gharbi A, Humblot V, Turpin F, Pradier CM, Imbert C, Berjeaud JM. 2012. Elaboration of antibiofilm surfaces functionalized with antifungal-cyclodextrin inclusion complexes. FEMS Immunol Med Microbiol. 65:257-269. doi: 10.1111/j.1574695X.2012.00932.x.

Ghosh M, Manivannan J, Sinha S, Chakraborty A, Mallick SK, Bandyopadhyay M, Mukherjee A. 2012. In vitro and in vivo genotoxicity of silver nanoparticles. Mutat Res
Genet Toxicol Environ Mutagen. 749:60-69. doi: 10.1016/j. mrgentox.2012.08.007.

Gilmore BF, Hamill TM, Jones DS, Gorman SP. 2010. Validation of the CDC biofilm reactor as a dynamic model for assessment of encrustation formation on urological device materials. J Biomed Mater Res B Appl Biomater. 93:128-140

Goeres DM, Loetterle LR, Hamilton MA, Murga R, Kirby DW, Donlan RM. 2005. Statistical assessment of a laboratory method for growing biofilms. Microbiology. 151:757-762. doi: 10.1099/mic.0.27709-0.

Heydorn A, Nielsen AT, Hentzer M, Sternberg C, Givskov M, Ersbøll BK, Molin S. 2000. Quantification of biofilm structures by the novel computer program comstat. Microbiology. 146:2395-2407. doi: 10.1099/00221287-146-10-2395.

Hall-Stoodley L, Costerton JW, Stoodley P. 2004. Bacterial biofilms: from the natural environment to infectious diseases. Nat Rev Microbiol. 2:95-108. doi: 10.1038/ nrmicro821.

Lagonenko L, Lagonenko A, Evtushenkov A. 2013. Impact of salicylic acid on biofilm formation by plant pathogenic bacteria. J Biol Earth Sci. 3:B176-B181.

Lo J, Lange D, Chew BH. 2014. Ureteral stents and Foley catheters-associated urinary tract infections: the role of coatings and materials in infection prevention. Antibiotics. 3:87-97. doi: 10.3390/antibiotics3010087.

Mangwani N, Shukla SK, Kumari S, Das S, Rao TS. 2016. Effect of biofilm parameters and extracellular polymeric substance composition on polycyclic aromatic hydrocarbon degradation. Rsc Adv. 6:S7540-S7551.

McDonnell G, Russell AD. 1999. Antiseptics and disinfectants: activity, action, and resistance. Clin Microbiol Rev. 12:147179.

Muller E, Al-Attar J, Wolff AG, Farber BF. 1998. Mechanism of salicylate-mediated inhibition of biofilm in Staphylococcus epidermidis. J Infect Dis. 177:501-503. doi: 10.1086/ jid.1998.177.issue-2.

Nowatzki PJ, Koepsel RR, Stoodley P, Min K, Harper A, Murata H, Donfack J, Hortelano ER, Ehrlich GD, Russell AJ. 2012. Salicylic acid-releasing polyurethane acrylate polymers as anti-biofilm urological catheter coatings. Acta Biomater. 8:1869-1880. doi: 10.1016/j.actbio.2012.01.032.

Pechook S, Sudakov K, Polishchuk I, Ostrov I, Zakin V, Pokroy B, Shemesh M. 2015. Bioinspired passive anti-biofouling surfaces preventing biofilm formation. J Mat Chem B. 3:1371-1378. doi: 10.1039/C4TB01522C

Polo A, Foladori P, Ponti B, Bettinetti R, Gambino M, Villa F, Cappitelli F. 2014. Evaluation of zosteric acid for mitigating biofilm formation of Pseudomonas putida isolated from a membrane bioreactor system. Int J Mol Sci. 15:9497-9518. doi: 10.3390/ijms15069497.

Rabin N, Zheng Y, Opoku-Temeng C, Du Y, Bonsu E, Sintim HO. 2015. Biofilm formation mechanisms and targets for developing antibiofilm agents. Future Med Chem. 7:493512. doi: $10.4155 / \mathrm{fmc} .15 .6$.

Raj B, Udaya Sankar K, Siddaramaiah. 2004. Low density polyethylene/starch blend films for food packaging applications. Adv Polym Technol. 23:32-45. doi: 10.1002/ (ISSN)1098-2329.

Rosenberg LE, Carbone AL, Romling U, Uhrich KE, Chikindas ML. 2008. Salicylic acid-based poly(anhydride esters) for 
control of biofilm formation in Salmonella enterica serovar Typhimurium. Lett Appl Microbiol. 46:593-599. doi: 10.1111/j.1472-765X.2008.02356.x.

Sastri VR. 2010. Commodity thermoplastics: polyvinyl chloride, polyolefin, and polystyrene. In: Sastri VR, editor. Plastics in medical devices: properties, requirements, and applications. Amsterdam (NL): Elsevier Inc; p. 73-119.

Siddiqa AJ, Chaudhury K, Adhikari B. 2015. Hydrophilic low density polyethylene (LDPE) films for cell adhesion and proliferation. RRJOM. 1:43-54.

Simões M, Simões LC, Vieira MJ. 2010. A review of current and emergent biofilm control strategies. LWT - Food Sci Technol. 43:573-583. doi: 10.1016/j.lwt.2009.12.008.

Sobieh SS, Kheiralla ZMH, Rushdy AA, Yakob NAN. 2016. In vitro and in vivo genotoxicity and molecular response of silver nanoparticles on different biological model systems. Caryologia.69:147-161.doi:10.1080/00087114.2016.1139416.

Sousa ACA, Pastorinho MR, Takahashi S, Tanabe S. 2014. History on organotin compounds, from snails to humans. Environ Chem Lett. 12:117-137. doi: 10.1007/s10311-013-0449-8.

Stanley MS, Callow ME, Perry R, Alberte RS, Smith R, Callow JA. 2002. Inhibition of fungal spore adhesion by zosteric acid as the basis for a novel, nontoxic crop protection technology. Phytopathology. 92:378-383. doi: 10.1094/ PHYTO.2002.92.4.378.

Ventola CL. 2015. The antibiotic resistance crisis: part 1: causes and threats. Pharmacol Ther. 40:277-283.
Vila J, Soto SM. 2012. Salicylate increases the expression of marA and reduces in vitro biofilm formation in uropathogenic Escherichia coli by decreasing type 1 fimbriae expression. Virulence. 3:280-285. doi: 10.4161/viru.19205.

Villa F, Albanese D, Giussani B, Stewart PS, Daffonchio D, Cappitelli F. 2010. Hindering biofilm formation with zosteric acid. Biofouling. 26:739-752. doi: 10.1080/08927014.2010.511197.

Villa F, Pitts B, Stewart PS, Giussani B, Roncoroni S, Albanese D, Giordano C, Tunesi M, Cappitelli F. 2011. Efficacy of zosteric acid sodium salt on the yeast biofilm model Candida albicans. Microb Ecol. 62:584-598. doi: 10.1007/ s00248-011-9876-x.

Villa F, Villa S, Gelain A, Cappitelli F. 2013. Sub-lethal activity of small molecules from natural sources and their synthetic derivatives against biofilm forming nosocomial pathogens. Curr Top Med Chem. 13:3184-3204. doi: $10.2174 / 15680266113136660225$.

von Eiff C, Jansen B, Kohnen W, Becker K. 2005. Infections associated with medical devices: pathogenesis, management and prophylaxis. Drugs. 65:179-214. doi: 10.2165/00003495200565020-00003.

Williams DL, Woodbury KL, Haymond BS, Parker AE, Bloebaum RD. 2011. A modified CDC biofilm reactor to produce mature biofilms on the surface of peek membranes for an in vivo animal model application. Curr Microbiol. 62:1657-1663. doi: 10.1007/s00284-011-9908-2. 